\title{
PERCEPCIÓN DE LOS EFECTOS VIVENCIALES DEL CAMBIO CLIMÁTICO EN UNA MUESTRA DE HABITANTES URBANOS AUSTRALES
}

\author{
CAROLINA NOVIÓN AICÓN ${ }^{*}$ Y CLAUDIA ESTRADA GOIC"*
}

\begin{abstract}
RESUMEN
El cambio climático y sus efectos a corto y largo plazo sobre la vida humana, es uno de los temas que ha despertado mayor interés en diferentes disciplinas científicas. Aunque en el seno de la psicología no existen investigaciones sobre esta temática, resulta evidente que los cambios en el ambiente tienen impacto sobre el bienestar de las personas. El presente estudio procura medir la percepción de cambio climático y el bienestar psicológico, y establecer la existencia de una relación entre ambas variables. La investigación consiste en un estudio no experimental transversal correlacional, donde la muestra no probabilística accidental estuvo compuesta por 120 habitantes de la ciudad de Punta Arenas, a quienes se les aplicaron cuestionarios midiendo la percepción de la existencia y magnitud del cambio climático en la vida cotidiana, y el bienestar psicológico. Los resultados indican que existen dos grupos de perceptores, aquellos que perciben la presencia de cambio climático y aquellos que no. Además, dentro de aquellos que dicen percibir estos cambios, se encuentran los que los valoran positivamente para su vida cotidiana y aquellos que los vinculan con aspectos negativos. Finalmente, los datos muestran la existencia de una vinculación entre estas variables únicamente para el dominio "subjetivo" y para la dimensión de "dominio del entorno"; quienes presentan mayor percepción de cambio climático, sin importar si este es evaluado como positivo o negativo, presentan también una disminución de su bienestar psicológico.
\end{abstract}

PALABRAS CLAVE: percepción, cambio climático, bienestar psicológico.

* Facultad de Humanidades, Ciencias Sociales y Ciencias de la Salud. Escuela de Psicología Universidad de Magallanes, Punta Arenas, Chile

* Psicología, Universidad de Magallanes, Av. Bulnes 01855, Punta Arenas, Chile. claudia.estrada@umag.cl 


\title{
THE PERCEPTION OF EXPERIENTIAL EFFECTS OF CLIMATE CHANGE IN A SAMPLE OF SOUTHERN URBAN POPULATION
}

\begin{abstract}
Climate change and its effects in the short and long term life human, is a topic that has attracted the most interest in different scientific disciplines. Even within psychology does not exist research on this subject, it is clear that changes in the environment have an impact on the welfare of people. The this study attempts to measure the perception of climate change and Psychological wellbeing and establish existence of a relationship between both variables. The research consists of a non-experimental cross correlation, where the probability accidental sample consisted of 120 inhabitants of the city of Punta Arenas, who were administered questionnaires measuring the perceived existence and magnitude of climate change in everyday life, and psychological wellbeing. The results indicate that there are two groups recipients, those who perceive the presence of climate change those who do not. Moreover, within those who claim to perceive these changes are the positive values for life daily and those that associate with negative aspects. Finally, the data show the existence of a link between these variables domain only for "subjective" and the dimension of "domain environment ", those with higher perception of climate change, without whether this is assessed as positive or negative, have also a decrease in psychological well-being.
\end{abstract}

KEY WORDS: perception, climate change, psychological well-being.

\section{INTRODUCCIÓN}

El fenómeno del cambio climático nos resulta cada vez más familiar. Las investigaciones en esta temática han trascendido súbitamente los laboratorios llegando con mucha frecuencia a la opinión pública, aunque en ocasiones de manera distorsionada. Según algunos investigadores (Camarasa y Moreno, 1994), los medios de comunicación poco rigurosos han contribuido a crear una imagen catastrófica del hecho siendo que aún los cambios no son percibidos por la población con tanta magnitud como se señala. Tal como lo expresa Retamales (2009), el conocimiento de los especialistas resulta inútil si este no llega de la manera adecuada al público en general.

Usar el término "Cambio Climático" en referencia a los cambios ocurridos recientemente en la Tierra resulta confuso, considerando que el estudio del pasado demuestra que el clima ha cambiado constantemente y desde antes que existiéramos los humanos. Por lo que resulta trascendente mencionar que las variaciones en el clima pueden ser el producto natural de fenómenos internos del sistema Tierra-atmósfera o pueden ser causadas por fuerzas externas (variaciones de la órbita terrestre, en la cantidad de energía solar) y sólo más recientemente la actividad humana se ha convertido en otro factor modificador del clima (Staines, 2007-08). Otros investigadores indican que más de un 90\% de las causas de este hecho son atribuibles a la acción del hombre, quienes serían responsables de una intensificación del "efecto invernadero" (Cerda, Valdivia, Valenzuela y Venegas, 2008), el cual consiste en un aumento de las concentraciones atmosféricas (como dióxido de carbono, metano y óxido nitroso) debido a la quema de combustibles fósiles, una progresiva deforestación, etc., todo esto marcadamente después de la Revolución Industrial.

Sea cual sea la causa o las causas del cambio climático, las evidencia indican que las temperaturas promedio mundiales aumentaron en más de $0,5^{\circ} \mathrm{C}$ en el último siglo, siendo la década del 90 una de las más cálidas registradas hasta el momento (Menghi, 2007). Dentro del debate que se ha llevado a cabo, la comunidad científica mundial piensa, mayoritariamente, que existe un aumento paulatino de la temperatura del clima en nuestro planeta, donde han proyectado un alza de la temperatura promedio planetaria de $1,0^{\circ} \mathrm{C}$ a $3,5^{\circ} \mathrm{C}$ para el año 2100 (Cerda et al., 2008). Además mencionan que las probables consecuencias de estos cambios son numerosas y poco alentadoras, donde encontramos 
un aumento en la demanda de energía para la refrigeración, disminución de la calidad del aire en las ciudades, aumento en demanda por agua, aumento en riesgo de mortalidad relacionado con el calor, áreas afectadas por el aumento de las sequías; hasta episodios de precipitación intensa en otros sectores, entre otras. El cambio climático, entendido como debido a actividades humanas, compromete la sostenibilidad del desarrollo humano amenazando los sistemas de apoyo ecológico de los que dependen la vida, la salud y el bienestar de toda la humanidad (Martens et al., 1998).

Con este panorama tan poco alentador para el futuro, comunicado por los medios masivos de información, las personas son llevadas, inevitablemente, a reflexionar acerca de su propia vida y de los efectos que estos cambios podrían estar teniendo en su calidad de vida presente y futura.

El concepto de calidad de vida surgió originalmente en la sociología con el propósito de dar cuenta de las diferencias entre los diversos recursos que cada persona posee y usa, y la valoración que esta persona hace de estos bienes y recursos (Giner et al., 2001). En el seno de la psicología ha recibido diferentes definiciones. Una de las consideradas como integradoras es la propuesta por Ardila (2003 en Gallardo y Salazar, 2010) quien la define como un estado de satisfacción general que sería el resultado de la actualización de las potencialidades que posee un individuo. Como tal, posee tanto aspectos objetivos como subjetivos. Los objetivos incluirían el bienestar material, las relaciones con el ambiente físico y social, con la comunidad y la salud física. En su concepción más amplia, la calidad de vida recibe la influencia de factores como empleo, vivienda, acceso a servicios públicos, comunicaciones, urbanización, criminalidad, contaminación del ambiente y otros que conforman el entorno social y que influyen sobre el desarrollo humano de una comunidad (VelardeJurado, 2002). Los subjetivos, en tanto, serían una sensación interna de bienestar físico, psicológica y social. Aunque ambos han mostrado importancia las Naciones Unidas, en su programa para el desarrollo humano, ha privilegiado la medición de los indicadores objetivos mientras que la psicología social se ha hecho cargo de la medición de los aspectos subjetivos de este fenómeno. Sin embargo de lo anterior, la calidad de vida, en ambos aspectos, incluye un amplio rango de hechos tales como el bienestar en diferentes dominios de la vida, en la salud, la situación financiera, el trabajo, el ocio, la vivienda y el medio ambiente.

Un concepto frecuentemente asociado a la dimensión subjetiva de calidad de vida en psicología es el denominado "bienestar subjetivo", el que ha sido verificado como fuertemente correlacionado con la satisfacción vital, es decir, con la evaluación cognitiva más general acerca de la propia vida. La investigación en psicología sobre bienestar se ha enfocado desde dos perspectivas fundamentales. Por un lado la tradición hedónica del bienestar se basa en la idea que la felicidad y el placer son sus bases fundamentales. Por otro lado la tradición eudamónica para la cual la felicidad se produce por la realización de tareas que permiten el crecimiento del ser humano como algo diferente a la búsqueda del placer por el placer (Diener et al., 1999). De esta última perspectiva Ryff y sus colaboradores (Keyes et al., 2002) proponen el concepto de bienestar psicológico el que sería logrado gracias al logro de metas o superación de desafíos existenciales. Así entonces algunos autores han extendido los límites de esta clasificación y han utilizado el constructo bienestar subjetivo (subjective well-being, SWB) como el principal de la tradición hedónica, y el constructo bienestar psicológico (psychological well-being, PWB) como el principal de la tradición eudamónica.

Mientras desde la tradición del bienestar subjetivo se ha mostrado especial interés en el estudio de los afectos y la satisfacción con la vida, en la correspondiente al bienestar psicológico, la atención ha estado centrada en el desarrollo de las capacidades y el crecimiento personal. Buscando los puntos de convergencia entre estas dos grandes formulaciones, Ryff (1989b) sugirió un modelo multidimensional de bienestar psicológico compuesto por seis dimensiones: autoaceptación, relaciones positivas con otras personas, autonomía, dominio del entorno, propósito en la vida, y crecimiento personal.

La autoaceptación es definida como una característica fundamental de la salud mental y del crecimiento personal, del funcionamiento óptimo y la madurez (Diaz et al., 2006; Ryff, 1989a) se relaciona a nivel individual de manera muy profunda con el autoconocimiento (Ryff y Keyes, 1995) y la manera en que las personas se evalúan a sí mismas. La dimensión de Relaciones Positivas se refiere a 
la necesidad que tienen las personas de mantener relaciones positivas con otras personas que sean estables y basadas en confianza (Baumeister y Leary, 1995). Esta necesidad, se fundamenta en el deseo de ser aceptados socialmente, un aspecto central de la autoestima. De este modo se configura parte de la identidad de la persona, en la medida en que permite tanto establecer semejanzas, como diferencias con otros (Turner, 1990). La dimensión de Autonomía menciona que para poder sostener su propia individualidad en diferentes contextos sociales, las personas necesitan asentarse en sus convicciones y mantener su independencia y autoridad personal. Cuando se posee autonomía las personas son capaces de resistir en mayor medida la presión social y auto-regulan mejor su comportamiento (Baumeister, 1998; Ryan y Deci, 2001; Ryff y Singer 2002; Snyder, 1974). La dimensión Dominio del Entorno señala que la habilidad para elegir o crear entornos favorables para satisfacer los deseos o necesidades propias otorga una mayor sensación de control sobre el mundo. Poseer una sensación de control tiene consecuencias muy positivas para las personas, ya que por ejemplo, permite reducir el estrés en entornos de trabajo y de estudio, pues las personas perciben que tienen los recursos necesarios para satisfacer las demandas del entorno (De Miguel et al., 2009; Karasek; 1979). En cambio la falta de control lleva a que las personas se sometan a entornos que paulatinamente pueden transformarse en tensionantes y amenazantes. El Crecimiento Personal es la dimensión que refiere al esfuerzo de las personas por buscar el desarrollo de sus propias potencialidades, para madurar y lograr el máximo de sí mismo (Ryff, 1989a). Según Rogers (1974), la búsqueda principal de las personas es hacia aquello que ellas mismas son, es decir su "yo real". La dimensión Propósito en la Vida habla de que el bienestar psicológico se configura también a partir de la búsqueda y logro de objetivos de vida; metas que permitan a las personas dotar a su existencia de un cierto sentido (Ryff, 1989b).

La evidencia señala que el conjunto de estas dimensiones no se dan de forma aislada sino que interactúan con contextos más amplios, como lo son los contextos microsocial y macrosocial, de esta forma emerge el concepto de dominios de satisfacción, dentro de las cuales se suele identificar el área laboral, el área de las relaciones interpersonales, el área material y el área subjetiva (Sanchez-Cánovas, 1998).

Resumiendo, podemos decir que el bienestar psicológico se lograría gracias al logro de metas o a la superación de desafíos existenciales, asociado principalmente a la autoaceptación, el establecimiento de relaciones positivas, la autonomía, el dominio del entorno, los propósitos en la vida y el crecimiento personal, que pueden darse dentro de los dominios laboral, material, subjetivo y relaciones interpersonales.

Cuando pensamos en la relación entre el cambio climático y el bienestar psicológico, podemos hipotetizar, que al comunicarnos que el calentamiento global acabará lentamente con la vida planetaria en el futuro, existirán diversas reacciones posibles: Algunas personas que se alarmaron con el tema y que se ven afectados en alguna de las dimensiones o dominios existentes en el bienestar de cada uno de los sujetos, por tal, comienzan a prepararse para tal acontecimiento, otros intentando alargar el proceso y sus efectos, y otros tratando de evitar que suceda tal panorama, ya sea utilizando productos que no destruyan la capa del ozono, reciclando la basura u otras actividades que lo convenzan acerca de la prevención. Con estos mínimos actos, que en el pasado no realizaban y de los cuales ahora tomaron como un hábito, han modificado su rutina y su forma de vivir, ya que optaron por tener otra preocupación de la cual no estaban acostumbrados $y$ es por esto que al sumarle otra inquietud a su vida cotidiana vamos a ver que su bienestar psicológico se ve afectado. El objetivo de la presente investigación es recoger el aspecto subjetivo del fenómeno desde dos perspectivas: ¿Perciben los puntarenenses diferencias en su entorno ambiental atribuibles al cambio climático? ¿Piensan que estos cambios los afectan positiva o negativamente?, ¿esta percepción negativa o positiva, se asocia a diferentes niveles de bienestar psicológico? Las respuestas a estas preguntas no sólo nos permitirá conocer la representación de este fenómeno a nivel local, sino que nos permitirá aportar a la comprensión de las variables implicadas en la movilización de las masas para el cambio social y la responsabilidad común ante hechos ambientales y ecológicos, y es debido a esto último que la influencia del cambio climático en el bienestar psicológico de los sujetos cobra importancia. 


\section{MÉTODO}

\section{Participantes y Diseño}

La muestra, no probabilística accidental, estuvo compuesta por 120 participantes adultos todos residentes en la ciudad de Punta Arenas. Un $52,5 \%$ fueron de género femenino y $47,5 \%$ de género masculino, con un rango de edad entre los 16 a los 74 años. Se utilizó un diseño no experimental, transversal correlacional.

\section{Instrumentos}

Instrumento de Percepción del Impacto del Cambio Climático: Su objetivo es determinar la actitud de los participantes frente al cambio climático. Compuesto por dos partes, la primera de ellas evalúa si los participantes perciben o no cambios en el clima de la región en áreas tales como: Nivel de radiación solar, lluvias, nieve y escarcha, viento, luz, temperaturas en verano, temperaturas en invierno, nivel del mar y derretimiento de los hielos. La segunda parte, mide si el cambio percibido es caracterizado como positivo o negativo a partir de los efectos que tiene en su vida cotidiana y la fuerza de dicho impacto. Se evaluó el impacto percibido en: estado de ánimo, energía para realizar actividades, actividades al aire libre, siembra de hortalizas, siembra o cultivo de jardines, vestuario que se utiliza, cuentas de la luz, agua y gas, estado de salud en general, alimentación, estudios / educación, trabajo, relaciones sociales, sueño y descanso. Se utilizaron escalas Likert (a: percibo una gran disminución hasta e: percibo un gran aumento), preguntas de alternativas múltiples (me afecta positivamente, me afecta negativamente, no me afectan) y medidas de porcentaje. La validez de contenido fue evaluada mediante la técnica de jueces expertos. La confiabilidad fue pretesteada (prueba piloto, $\mathrm{n}=10$ )

Cuestionario de bienestar Psicológico (Estrada y Avendaño, 2009): Cuestionario que mide el nivel de bienestar psicológico que las personas experimentan respecto de sus vidas. Fue construido integrando dos modelos distintos de bienestar; dominios de bienestar: subjetivo, laboral, relaciones interpersonales y material; y dimensiones de bienestar: control de situaciones, aceptación de sí mismo, vínculos psicosociales, proyectos, autonomía y felicidad (Díaz,
Rodríguez et al., 2006). El instrumento compuesto por 44 ítems, en el que cada afirmación mide un dominio y una dimensión simultáneamente que se responden en base a una escala Likert de 5 puntos que va desde el 1:"Nunca" hasta el 5:"Siempre". El instrumento presentó una confiabilidad de 0,92 (Alfa).

\section{Procedimiento}

La aplicación de ambos instrumentos fue de forma individual (autoaplicada) y voluntaria. Inicialmente se les informó sobre los objetivos generales del estudio y firmaron un consentimiento de participación. Finalmente se les agradeció por su participación y se les ofreció un informe de sus resultados individuales.

\section{RESULTADOS}

\section{Percepción de cambio}

Al analizar la información descriptiva arrojada por el Instrumento de Medición de Cambio Climático, encontramos que respecto a la percepción que tienen los habitantes de Punta Arenas sobre la presencia de indicadores de alteraciones sensibles, estos se encuentran para 7 de las 9 características evaluadas (ver tabla 1). El área que presenta mayor percepción de cambio es " nieve y escarcha" con un $89,2 \%$ donde la mayoría indica que este cambio va en la dirección de una disminución de la nieve y la escarcha (91,6\%), mientras que el porcentaje restante indica un aumento (8,4\%). Sólo un 10,8\% de los encuestados considera que no percibe cambios sobre esta área.

Otro de los factores que presenta una alta percepción de cambio climático es la "Temperatura en Verano" con un 73,3\% donde la mayor parte de ellos percibe un aumento en las temperaturas (75\%). Cuando se trata de "la radiación solar", el 71,7\% percibe variaciones, la gran mayoría inclinándose también por un aumento (97,6\%). En cuanto a las "lluvias" podemos observar que el 70\% de la muestra percibe algún tipo de cambio, gran parte de ellos coincide en que se trata de un aumento en los niveles de agua caída (75\%). De forma similar, el 57,7\% perciben cambios en los "Derretimientos de los Hielos", donde la mayoría indica que han aumentado $(76,6 \%)$. 
Tabla 1. Percepción de cambio climático en la Región de Magallanes en porcentajes.

\begin{tabular}{|c|c|c|c|}
\hline Área de cambio & Percepción de disminución & Percepción de aumento & No percibe cambios \\
\hline 1. Nieve y la escarcha & $81,7 \%$ & $7,5 \%$ & $10,8 \%$ \\
\hline 2. Temperaturas en verano & $18,3 \%$ & $55,0 \%$ & $26,7 \%$ \\
\hline 3. Radiación solar & $1,7 \%$ & $70,0 \%$ & $28,3 \%$ \\
\hline 4. Lluvias & $17,5 \%$ & $52,5 \%$ & $30,0 \%$ \\
\hline 5. Derretimiento de los hielos & $13,3 \%$ & $44,2 \%$ & $42,5 \%$ \\
\hline 6. Temperaturas en invierno & $28,3 \%$ & $28,3 \%$ & $43,4 \%$ \\
\hline 7. Viento & $10,0 \%$ & $40,0 \%$ & $50,0 \%$ \\
\hline 8. Luz & $19,2 \%$ & $7,5 \%$ & $73,3 \%$ \\
\hline 9. Nivel del mar & $1,7 \%$ & $11,6 \%$ & $86,7 \%$ \\
\hline
\end{tabular}

Sobre las "temperaturas en invierno", la percepción de cambio alcanza el 56,6\% que se reparte entre un aumento de la temperatura (50\%) y una disminución (50\%) en ésta época del año y con un $43,3 \%$ de la muestra que no percibe cambios.

Finalmente nos encontramos con que existe sólo un 50\% de la muestra, que percibe algún tipo de cambio en la variable "viento" (aumento $=40 \%$ y disminución $=10 \%)$.

En cuanto a la variable "luz" (73,3\%) y "niveles del mar" (86,7\%), la mayoría de los participantes no percibe cambios perceptibles en estas áreas.

Percepción de efecto en la vida cotidiana

Con relación a cómo perciben los habitantes de Punta Arenas que les afectan el cambio climático en su vida cotidiana, nuevamente "la nieve y escarcha" toma la primera posición, para indicarnos que un $76,6 \%$ de los encuestados cree que esta alteración en el clima le afecta en su vida diaria, ya sea positiva $(59,8 \%)$ o negativamente (40,2\%), el 23,3\% restante considera que los cambios percibidos no afectan su diario vivir. A continuación encontramos la "Temperatura en verano" con un 76,6\%, donde la mayoría coincide en que los afecta negativamente (66,3\%), la "radiación solar", donde un 70\% de los participantes coincide en que esta área afecta su vida cotidiana $(90,4 \%$, de ellos opina que de forma negativa). Un 67,5\% indica que los cambios en las "temperaturas en invierno" los afectan (un 69,2\% de forma positiva), un 63,3\% cuando se trata de la "Lluvia" (un 92\% de forma positiva) y un 53,3\% al referirse al "viento" (89\% de forma positiva).

Finalmente, existe una menor percepción de que puedan afectar en la vida cotidiana de las personas que residen en Punta Arenas los cambios en: "la luz" con un 59,2\%, el "derretimiento de los hielos" con un 63,3\% y el "nivel del mar" con un $87,5 \%$ que considera que no afecta en su vida diaria (ver tabla 2).

Respecto al efecto que los cambios percibidos tienen en diferentes aspectos de la vida personal y comunitaria, es opinión de los participantes que su efecto tiende a ser negativo. Es decir, la mayoría de los cambios percibidos en el clima estarían perturbando

Tabla 2. Cómo afecta el cambio climático en la vida cotidiana de los habitantes de Punta Arenas en porcentajes.

\begin{tabular}{lccc}
\hline \multicolumn{1}{c}{ Área de cambio } & $\begin{array}{c}\text { Afecta } \\
\text { positivamente }\end{array}$ & $\begin{array}{c}\text { Afecta } \\
\text { negativamente }\end{array}$ & No afecta \\
\cline { 1 - 3 } \cline { 3 - 4 } 1. Nieve y la escarcha & $45,8 \%$ & $30,8 \%$ & $23,3 \%$ \\
2. Temperaturas en verano & $25,8 \%$ & $50,8 \%$ & $23,3 \%$ \\
3. Radiación solar & $6,7 \%$ & $63,3 \%$ & $30,0 \%$ \\
4. Temperaturas en invierno & $46,7 \%$ & $20,8 \%$ & $32,5 \%$ \\
5. Lluvias & $58,3 \%$ & $5,0 \%$ & $36,7 \%$ \\
6. Viento & $47,5 \%$ & $5,8 \%$ & $46,7 \%$ \\
7. Luz & $32,5 \%$ & $8,3 \%$ & $59,2 \%$ \\
8. Derretimientos de los hielos & $32,5 \%$ & $4,2 \%$ & $63,3 \%$ \\
9. Nivel del mar & $11,7 \%$ & $0,8 \%$ & $87,5 \%$ \\
\hline
\end{tabular}


Tabla 3. Porcentajes de percepción del efecto positivo o negativo del cambio climático por áreas afectadas.

\begin{tabular}{|c|c|c|c|c|c|c|c|c|c|c|c|c|}
\hline \multirow[t]{2}{*}{$\%$} & \multicolumn{2}{|c|}{ Radiación solar } & \multicolumn{2}{|c|}{ Lluvias } & \multicolumn{2}{|c|}{ Nieve y escarcha } & \multicolumn{2}{|c|}{ Viento } & \multicolumn{2}{|c|}{$\begin{array}{c}\text { Temperatura en } \\
\text { verano }\end{array}$} & \multicolumn{2}{|c|}{$\begin{array}{c}\text { Temperatura en } \\
\text { invierno }\end{array}$} \\
\hline & Positivo & Negativo & Positivo & Negativo & Positivo & Negativo & Positivo & Negativo & Positivo & Negativo & Positivo & Negativo \\
\hline $\begin{array}{l}\text { Actividades aire } \\
\text { libre }\end{array}$ & 10,0 & 63,3 & 6,7 & 74,2 & 21,7 & 55,0 & 6,7 & 66,7 & 53,3 & 20,8 & 20,0 & 55,0 \\
\hline $\begin{array}{l}\text { Estado de salud } \\
\text { en general }\end{array}$ & 4,2 & 55,0 & 2,5 & 51,7 & & 45,8 & 2,5 & 28,3 & 30,8 & 12,5 & 14,2 & 47,5 \\
\hline $\begin{array}{l}\text { Estado de } \\
\text { ánimo }\end{array}$ & & & & & & & 6,7 & 38,3 & 53,3 & 20,0 & 18,3 & 45,8 \\
\hline $\begin{array}{l}\text { Energía realizar } \\
\text { actividades }\end{array}$ & 10,8 & & 6,7 & & & & 7,5 & 48,3 & 51,7 & 19,2 & 18,3 & 48,3 \\
\hline Vestuario utiliza & 10,8 & 36,7 & 6,7 & 52,5 & 20,8 & 35,0 & 4,2 & 32,5 & 45,8 & 13,3 & 16,7 & 41,7 \\
\hline
\end{tabular}

la calidad de vida de los habilitantes de la ciudad. La variable que afecta un mayor número de actividades, todas ellas de forma negativa, es la "lluvia" alcanzando a: las actividades al aire libre (74,2\%), el estado de ánimo (58,3\%), la energía (58,3\%), el vestuario que se utiliza $(52,5 \%)$ y el estado de salud en general $(51,7 \%)$ afectando negativamente en su quehacer. La variable "radiación solar" afecta negativamente: las actividades al aire libre $(63,3 \%)$ y el estado de salud en general (55\%). El "viento" (66,7\%), la " nieve y escarcha" (55\%), y las "temperaturas en invierno" (55\%) afectan negativamente las actividades al aire libre que se puedan realizar en la región. Finalmente encontramos que la variable "temperaturas en Verano" afecta de manera positiva: el estado de ánimo y las actividades al aire libre (53,3\%) y también la energía para realizar actividades $(51,7 \%)$ (ver Tabla 3). En cuanto a la variable "luz" no se encontraron actividades que fueran percibidas mayoritariamente como perturbadas por los cambios.

\section{Percepción de cambio y bienestar psicológico}

Al evaluar la relación entre la percepción de cambio climático y el bienestar psicológico es posible observar que ambas variables se encuentran asociadas únicamente en el dominio subjetivo y no en el laboral, material o de relaciones interpersonales. La asociación observada indica que el aumento de percepción de cambio climático se acompaña de una disminución en el bienestar subjetivo, $r_{(120)}=$ $-0.22, p<0,05$. Al tratarse de las dimensiones del bienestar (autoaceptación, relaciones positivas con otras personas, autonomía, dominio del entorno, propósito en la vida, y crecimiento personal), se observa una relación negativa entre la percepción de cambio climático y el dominio del entorno, $r_{(120)}=$ $-0.18, p<0,05$.

\section{DISCUSIÓN Y CONCLUSIONES}

La presente investigación tuvo como objetivo describir la percepción de cambio climático y el bienestar subjetivo que poseen los habitantes de la ciudad de Punta Arenas y la relación existente entre ambas variables. Los 120 habitantes de la ciudad que participaron como voluntarios, fueron medidos en percepción de cambio climático en las áreas de Niveles de radiación solar, las lluvias, La nieve y escarcha, el viento, la luz, las temperaturas en verano, las temperaturas en invierno, el nivel del mar y los derretimientos de los hielos.

Los resultados indican que los participantes aprecian cambios climáticos en un 78\% de las áreas propuestas. En particular, perciben diferencias detectables en la "nieve y escarcha", "temperaturas en verano", "radiación solar" y "lluvias", entre otros, con claro acuerdo de la dirección de estos cambios. Una excepción a esta regla es la situación de "temperaturas en invierno" ya que aunque la mayoría de los participantes dice haber percibido cambio, la mitad de ellos indica que es hacia la dirección de la disminución de las temperaturas mientras que el $50 \%$ restante opina lo contrario. El interés de este resultado es que revela la dimensión subjetiva de la percepción de cambios ya que la "vivencia objetiva" compartida por vivir en un mismo lugar, no es interpretada de la misma manera.

Los análisis sobre la percepción de los efectos de los cambios sobre la vida cotidiana, indican 
que para "radiación solar" existe una posición mayoritaria indicando que estos cambios resultan negativos para la población así como para "lluvia" que es mayoritariamente percibida como afectando positivamente. Las otras dimensiones evaluadas muestran posiciones menos claras en la que coexisten grupos que señalan que los afecta ya sea positiva o negativamente. Una vez más, la subjetividad define el significado de este fenómeno más que la vivencia "objetiva". Un alto porcentaje indicó que los aspectos de la vida personal que se encuentran dentro de los más afectados negativamente por los cambios son el estado de ánimo y la salud física en general. Ambas categorías resultan particularmente importantes ya que reflejan la creencia que la salud física y mental es susceptible de ser afectada por el entorno ambiental. En Chile, la tasa anual de suicidios durante el 2008 alcanzó a un 10,8 por 100.000. En Punta Arenas, esta tasa llegó a 23,8\%, superando con creces el promedio nacional. Tal y como lo señala Moyano (2010), el suicidio es un adecuado indicador de bienestar y de salud mental. Aunque este fenómeno se ha visto fuertemente conectado con la presencia de consumo de alcohol, también es cierto que factores estacionales han sido expuestos como responsables por expertos locales. Particularmente, se indica que la primavera conduce al acentuamiento de algunas psicopatologías y sus manifestaciones. Es posible, que una parte del fenómeno radique en las creencias que las personas poseen sobre las dificultades que enfrentan en su propia vida debido a su ambiente físico. Esta hipótesis explicativa, encuentra apoyo empírico en el resultado que indica la existencia de una relación entre la percepción de cambio y el bienestar psicológico. La hipótesis de investigación postuló que quienes presentan mayor percepción de cambio climático, tendrían un menor nivel de bienestar psicológico. Al momento de observar los resultados, estos apoyan dicha hipótesis solo parcialmente, debido a que el bienestar psicológico, que de acuerdo al instrumento utilizado se mide en dominios (subjetivo, laboral, material o de relaciones interpersonales) y dimensiones (autoaceptación, relaciones positivas con otras personas, autonomía, dominio del entorno, propósito en la vida, y crecimiento personal), muestra asociaciones con dos de ellos. La percepción de cambio climático se acompaña de la disminución del bienestar psicológico en su dominio subjetivo, es decir, no se encuentra conectado con algún ámbito en particular, ni con lo laboral ni material ni con las relaciones interpersonales, sino que se vincula con el bienestar "interno" o "sentido" por la propia persona. En otros términos, el cambio climático no es vinculado con un aspecto concreto del bienestar sino con el mundo interno de cada individuo. Igualmente, la dimensión que hace referencia el control de situaciones aparece relacionada con el bienestar. De las dimensiones evaluadas, esta dimensión presenta una particular coherencia con el fenómeno de cambio climático que hace referencia a la habilidad para generar entornos favorables para la satisfacción de las propias necesidades. Percibir un entorno cambiante, "descontrolado", fuera de las posibilidades de acción concreta de un habitante, se acompaña de una disminución del bienestar asociado a esta sensación de control. y es sólo a algunos de estos, a los cuales afecta de forma considerable. Por tanto, un aumento en la percepción de cambio climático significa una disminución del Bienestar Psicológico, pero sólo en el dominio subjetivo y en la dimensión que hace referencia al control de situaciones. En la actualidad el bienestar es más que la ausencia de malestar, se entiende como el funcionamiento y experiencia psicológica en su estado más optimo (Ryan y Deci, 2001). El aumento en la percepción de cambio climático hace que disminuya la habilidad individual para elegir o crear ambientes que se acomoden a sus condiciones psicológicas y físicas. Una baja en la sensación de control, hace que las personas perciban una disminución en los recursos que poseen para satisfacer las demandas de su entorno y la falta de control lleva a que las personas se sometan a entornos que paulatinamente puedan transformarse en tensionantes y amenazantes. Si no puede lidiar con las demandas de un lugar, entonces aumenta su estrés, se reduce su bienestar psicológico y aumenta el desgaste corporal y psíquico, además de los problemas de salud (Gun Kang et al., 2004 en Gallardo y Salazar, 2010).

En resumen, estos resultados nos señalan que la mayoría de los participantes se sienten afectados negativamente por los cambios percibidos en cada unos de ellos, afectando especialmente su bienestar subjetivo, el cual está determinado por la valoración que el sujeto hace de su propia vida en función del nivel de satisfacción que alcanza en las esferas o dominios más importantes de su vida (García - Viniegras y González, 2000). 
Que esta percepción influya en el dominio del entorno, nos dice que es necesario trabajar para aumentar su sensación de control y les haga sentirse capaz de influir sobre su entorno (Díaz et al., 2006). El trabajo que se realice en la prevención de las consecuencias a futuro provocadas por el calentamiento global debería considerar esta dimensión ya que puede ser la puerta de entrada al cambio actitudinal. Por otra parte, la relación entre el individuo y su entorno físico es una pregunta abierta. Estos primeros resultados parecen indicar que es relevante ocuparse de los vínculos que dichas variables pueden tener en la salud física y mental de las personas. Este primer estudio, con sus limitaciones obvias debido al tipo de muestra y su tamaño, son una primera pista para resolver como enfrentar adecuadamente en el futuro tanto el "calentamiento global objetivo" (Indicado, por ejemplo, en el semáforo solar que nos indica la radiación presente), como el "calentamiento global subjetivo" que puede manifestarse en problemáticas asociadas a la salud como también en resistencias a adoptar nuevos comportamientos saludables por la percepción de no tener control sobre nuestro entorno.

Futuras investigaciones se enriquecerían del contraste entre la percepción de cambio y la realidad, es decir, comparar la mirada de diferentes actores (actividades profesionales y personales diversas, conectadas y no con el ambiente natural) con las estadísticas de los cambios registrados durante los últimos años. También resultaría de interés incluir la variable edad y permanencia en la región como elementos determinantes de la percepción de cambio. Probablemente aquellos que han desarrollado su vida en la región tendrán una visión longitudinal menos influenciada por la información de los medios de comunicación que aquellos jóvenes cuya vivencia directa es breve para el desarrollo de este fenómeno.

\section{BIBLIOGRAFÍA}

BAUMEISTER, R. 1998. The self. En: Handbook of Social Psychology. Editores D.T. Gilbert, S.T. Fiske y G. Lindzey, 4(1):680-740. MacGraw-Hill. New York.

BAUMEISTER, R.F. y M.R. LEARY 1995. The need to belong: Desire for interpersonal attachments as a fundamental human motivation. Psychological Bulletin. 117:497-529.

CAMARASA, A. y F. MORENO 1994. Algunas Reflexiones sobre la Percepción del Cambio Climático en una muestra de Población Adulta de nivel Cultural Medio. Serie Gráfic. 4:127-132.

CERDA J., VALDIVIA G., VALENZUELA M. y VENEGAS Y. 2008. Cambio Climático y Enfermedades Infecciosas. Un Nuevo Escenario Epidemiológico. Revista Chilena de Infectología. 25:447-452. Santiago.

DE MIGUEL, J., I. GALLARDO, J. HORCAJO, A. BECERRA, P. AGUILAR y P. BRIÑOL 2009. El efecto del estrés sobre el procesamiento de mensajes persuasivos. Revista de Psicología Social. 24: 399-409.

DÍAZ, D., R. RODRÍGUEZ-CARVAJAL, B. MORENO-JIMÉNEZ, I. GALLARDO, C. VALLE y D. VAN DIERENDONCK 2006. Adaptación española de las escalas de bienestar psicológico de Ryff. Psicothema, 18: 572-577.

DIENER, E., E.M. SUH, R.E. LUCAS y H.L. SMITH 1999. Subjective well-being: Three decades of progress. Psychological Bulletin, 125:276-302.

ESTRADA, C. y S. AVENDAÑO 2009. Cuestionario de bienestar psicológico. Chile.

GALLARDO, I. y C. SALAZAR 2010. Bienestar psicológico en adolescentes escolarizados maulinos. En: Calidad de Vida y psicología en el bicentenario de Chile. E. Moyano (Ed.) Facultad de Psicología. Talca.

GARCÍA-VINIEGRAS, C. y I. GONZÁLEZ 2000. La Categoría Bienestar Psicológico. Su Relación con otras Categorías Sociales. Revista Cubana Medicina General Integral. 16(6):586-92.

GINER, J., E. IBÁÑEZ, S. CERVERA, A. SANMARTÍN y R. CABALLERO 2001. Experiencia Subjetiva y calidad de vida en la esquizofrenia. Actas Españolas de Psiquiatría. 29:233-242.

KARASK, R 1979. Job demands, job decision latitude and mental strain: Implications for job redesign. Administrative Science Quarterly, 24:285-306

KEYES, C., C. RYFF y D. SHMOTKIN 2002. Optimizing wellbeing: the empirical encounter of two traditions. Journal of Personality and Social Psychology, 82:1007-1022.

MARTENS, W., R. SLOOFF y E. JACKSON 1998. El Cambio Climático, la Salud Humana y el Desarrollo Sostenible. Revista Panamericana de Salud Pública. 4:100-105. Washington.

MENGHI, C. 2007. Calentamiento Global: El Riesgo Oculto para la Salud. Revista Argentina de Microbiología. 39:131-132.

MOYANO, E. 2010. Calidad de Vida y Psicología en el Bicentenario de Chile. Universidad de Talca, Facultad de Psicología. Talca.

RETAMALES, J. 2009. Cambio Climático en la Región de Magallanes y Antártica: Evidencias y Desafíos para el 
Futuro. Inauguración Coloquio Internacional. Universidad de Magallanes, Punta Arenas.

ROGERS, C.R. 1974. El proceso de convertirse en persona. Mi técnica terapéutica. Editorial Paidós, Buenos Aires.

RYAN, R. M. y E.L. DECI 2001. To be happy or to be selffulfilled: A review of research on hedonic and eudaemonic well-being. En: S. Fiske (Ed.), Annual Review of Psychology, 52:141-166.

RYFF, C. 1989a. Beyond Ponce de Leon and life satisfaction: New directions in quest of successful aging. International Journal of Behavioral Development, 12:35-55.

RYFF, C. 1989b. Happiness is everything, or is it? Explorations on the meaning of psychological well-being. Journal of Personality and Social Psychology, 57:1069-1081.

RYFF, C. y C. KEYES 1995. The structure of psychological well-being revisited. Journal of Personality and Social Psychology, 69:719-727.
RYFF, C. y B. SINGER 2002. From social structure to biology. En: Handbook of positive psychology. Editores C. Snyder y A. López, pp. 63-73. Oxford University Press. Londres.

STAINES, F. 2007-08. Cambio Climático: Interpretando el Pasado para entender el Presente. Ciencia Ergo Sum, 14(3):345-351.

SÁNCHEZ CÁNOVAS, J. 1998. EBP. Escala de bienestar psicológico. TEA Ediciones, Madrid.

SNYDER, M. 1974. Self monitoring of expressive behavior. Journal of Personality and Social Psychology. 30:526-537.

TURNER, J.C. 1990. Redescubrir el grupo social. Morata, Madrid.

VELARDE-JURADO, E. y C. ÁVILA-FIGUEROA 2002. Evaluación de la Calidad de Vida. Salud Pública México. 44:349-361. 